\section{Câncer e TEP}

A TEV é uma complicação comum em pacientes com câncer, sendo causa frequente de morbidade e mortalidade.(1) De todos os pacientes com TEV, 20\% têm câncer subjacente, enquanto a neoplasia maligna está presente em 26\% dos casos novos de TEV idiopática. ${ }^{(2)}$ Cerca de 10\% dos pacientes têm câncer diagnosticado subsequentemente ao episódio inicial de TEV. ${ }^{(3,4)}$ 0 período maior de risco está entre 6-12 meses do episódio inicial de TEV. As neoplasias malignas mais comumente associadas são as de pâncreas, cérebro, estômago e ovário, sendo que o melanoma, o câncer de mama ou o de próstata têm uma incidência menor. Câncer avançado e metastático tem uma maior incidência relativa de TEV.

É questionável a realização de exames mais complexos para a pesquisa da localização de tumor nesses pacientes, sendo a sobrevida média, nesses casos, de 12\% em 1 ano, devido à alta frequência da doença já se encontrar em estágio avançado. ${ }^{(5)}$ É controverso realizar uma extensa investigação com exames sofisticados (TC de tórax e abdômen, endoscopia digestiva alta e baixa, tomografia por emissão de pósitrons, etc.). ${ }^{(6,7)}$ Uma investigação aprofundada em pacientes com TEV idiopática somente deve ser realizada a partir de achados positivos na história clínica, exame físico, exames laboratoriais de rotina e radiografia de tórax.(B)

0 surgimento de TEV é um fator prognóstico desfavorável em pacientes com esse tipo de doença, pois eles têm um risco de TEV recorrente e de óbito (por qualquer causa) três vezes maior do que pacientes com TEV recorrente e sem malignidade. ${ }^{(8,9)}$ Um em cada 7 pacientes com câncer hospitalizados morre devido a TEP. ${ }^{(10,11)}$ Cirurgias para câncer produzem um maior risco de TEV e de tromboembolia do que cirurgias em pacientes sem essa moléstia. ${ }^{(10)} \mathrm{A}$ quimioterapia, principalmente se for associada à hormonioterapia, também acarreta um aumento do risco de TEV nos portadores de neoplasia maligna. Nestes últimos anos, tem sido relatada uma alta taxa de TEV em pacientes tratados com a combinação de quimioterapia e inibidores da angiogênese. ${ }^{(12,13)}$ Outro fator que deve ser observado é a presença de cateteres venosos centrais, que frequentemente são utilizados nesses indivíduos, ocasionando um maior risco de trombose venosa.
Alterações nos parâmetros de hemostasia ocorrem frequentemente em pacientes portadores de neoplasias malignas, incluindo o aumento do fator Vlla, do complexo trombina-antitrombina e de estimuladores dos fatores tissulares da coagulação sanguínea. ${ }^{(14)}$ Os mecanismos de trombose venosa associada ao câncer estão hoje centrados nas propriedades intrínsecas das células neoplásicas que levariam ao estado pró-trombótico. Essa hipercoagulabilidade parece ser devido à aumentada expressão celular de fator tissular e à elevação dos níveis de fator tissular em micropartículas na circulação sistêmica. Contribuem também a invasão de células neoplásicas na parede vascular e na estase venosa, determinada pela compressão vascular por tumores sólidos. Outros fatores, como os sítios primários dos tumores, as condições gerais dos pacientes, a presença de comorbidades, as intercorrências cirúrgicas e os tipos de tratamento, acrescentam riscos que determinam uma incidência elevada de TEV em pacientes com câncer.

Os pacientes com neoplasias malignas apresentam um aumento de risco para TEV; no entanto, o uso profilático de terapia antitrombótica acarreta um maior risco de sangramento nesses indivíduos. ${ }^{(15)}$ Os pacientes com indicação de procedimentos cirúrgicos de maior porte devem receber profilaxia com terapia anticoagulante.(B) ${ }^{(12)}$ Apesar da profilaxia não ser a rotina atual em pacientes que são tratados com quimioterapia em regime ambulatorial, estudos atuais sugerem um benefício com o uso de HBPM no domicílio naqueles pacientes com neoplasia metastática ou localmente avançada em programa de quimioterapia.(B) ${ }^{(16)}$

As HBPM são mais eficazes que os $A V K$ em pacientes com TEV e câncer, tanto pela menor recorrência dos episódios de trombose, quanto pela maior tendência de reduzir a mortalidade. 0 uso de HBPM deve ser a primeira escolha no tratamento de TEV, tanto para a fase aguda, quanto para a fase de manutenção.(A) ${ }^{(17)} 0$ tempo de anticoagulação dependerá do número de ciclos de quimioterapia (e de quais fármacos), do tipo de câncer e da previsibilidade da doença em atividade, contrabalançando esses com o risco de sangramento. Se possível, o anticoagulante deverá ser utilizado por pelo menos 6-12 meses, indefinidamente ou até a resolução do câncer. A recorrência de TEV é alta, mesmo na vigência do tratamento. Nessa situação, alguns 
estudos observacionais sugerem que doses mais altas de HBPM podem ser mais efetivas que as doses convencionais. ${ }^{(18)}$

\section{Referências}

1. Lee AY. Epidemiology and management of venous thromboembolism in patients with cancer. Thromb Res. 2003;110(4):167-72.

2. Wun T, White RH. Epidemiology of cancer-related venous thromboembolism. Best Pract Res Clin Haematol. 2009;22(1):9-23.

3. Prandoni P, lensing AW, Büller HR, Cogo A, Prins MH, Cattelan AM, et al. Deep-vein thrombosis and the incidence of subsequent symptomatic cancer. $\mathrm{N}$ Engl $\mathrm{J}$ Med. 1992;327(16):1128-33.

4. Chew HK, Wun T, Harvey D, Zhou H, White RH. Incidence of venous thromboembolism and its effect on survival among patients with common cancers. Arch Intern Med. 2006;166(4):458-64.

5. Pavic M, Debourdeau P, Aletti M, Farge-Bancel D, Rousset $\mathrm{H}$. Venous thromboembolism and cancer [Article in French]. Rev Med Interne. 2006;27(4):313-22.

6. Piccioli A, Lensing AW, Prins MH, Falanga A, Scannapieco GL, leran M, et al. Extensive screening for occult malignant disease in idiopathic venous thromboembolism: a prospective randomized clinical trial. J Thromb Haemost. 2004;2(6):884-9.

7. Monreal $M$, lensing AW, Prins $M H$, Bonet $M$, Fernández-Llamazares J, Muchart J, et al. Screening for occult cancer in patients with acute deep vein thrombosis or pulmonary embolism. J Thromb Haemost. 2004;2(6):876-81.

8. Lee AY, Levine MN. Venous thromboembolism and cancer: risks and outcomes. Circulation. 2003;107(23 Suppl 1):117-21.
9. Sørensen HT, Mellemkjaer L, Olsen JH, Baron JA. Prognosis of cancers associated with venous thromboembolism. N Engl J Med. 2000;343(25):1846-50.

10. Stein PD, Beemath A, Meyers FA, Skaf E, Sanchez J, Olson RE. Incidence of venous thromboembolism in patients hospitalized with cancer. Am J Med. 2006;119(1):60-8.

11. Heit JA, Silverstein MD, Mohr DN, Petterson TM, O'Fallon WM, Melton LJ 3rd. Risk factors for deep vein thrombosis and pulmonary embolism: a population-based casecontrol study. Arch Intern Med. 2000;160(6):809-15.

12. Behranwala KA, Williamson RC. Cancer-associated venous thrombosis in the surgical setting. Ann Surg. 2009;249(3):366-75.

13. Kakkar AK, Levine M, Pinedo HM, Wolff R, Wong J. Venous thrombosis in cancer patients: insights from the FRONTLINE survey. Oncologist. 2003;8(4):381-8.

14. Khorana AA. Cancer and thrombosis: implications of published guidelines for clinical practice. Ann Oncol. 2009;20(10):1619-30.

15. Ansell JE. Prophylaxis and treatment of venous thromboembolism in cancer patients: a review. Am J Clin Oncol. 2009;32(4 Suppl):S8-S12.

16. Agnelli G, Gussoni G, Bianchini C, Verso M, Mandalà $\mathrm{M}$, Cavanna L, et al. Nadroparin for the prevention of thromboembolic events in ambulatory patients with metastatic or locally advanced solid cancer receiving chemotherapy: a randomised, placebo-controlled, double-blind study. Lancet Oncol. 2009;10(10):943-9.

17. Imberti D, Di Nisio M, Donati MB, Falanga A, Ghirarduzzi A, Guarneri D, et al. Treatment of venous thromboembolism in patients with cancer: Guidelines of the Italian Society for Haemostasis and Thrombosis (SISET). Thromb Res. 2009;124(5):e32-40.

18. Carrier M, Le Gal G, Cho R, Tierney S, Rodger M, Lee AY. Dose escalation of low molecular weight heparin to manage recurrent venous thromboembolic events despite systemic anticoagulation in cancer patients. J Thromb Haemost. 2009;7(5):760-5. 